\title{
Tobacco Smoking Among Incarcerated Individuals: A Review of the Nature of the Problem and What is Being Done in Response
}

John J. Donahue

To cite this article: John J. Donahue (2009) Tobacco Smoking Among Incarcerated Individuals: A Review of the Nature of the Problem and What is Being Done in Response, Journal of Offender Rehabilitation, 48:7, 589-604, DOI: 10.1080/10509670903196058

To link to this article: http://dx.doi.org/10.1080/10509670903196058

舟 Published online: 09 Sep 2009.

Submit your article to this journal $\square$

Џ Article views: 64

Q View related articles ๔

Citing articles: 1 View citing articles 진 


\title{
Tobacco Smoking Among Incarcerated Individuals: A Review of the Nature of the Problem and What is Being Done in Response
}

\author{
JOHN J. DONAHUE \\ Department of Psychology, La Salle University, Philadelphia, Pennsylvania, USA
}

\begin{abstract}
Smoking is a major health problem, however the issue is even more pronounced among those incarcerated in prisons, where smoking rates are often three times that of the general population. While effective treatments have been demonstrated in the tobacco literature, research examining treatment within prisons is limited in scope. This article reviews the existing literature evaluating the extent of this problem, its demonstrated effects, and the responses by bealth care and correctional authorities. Overall, this problem is far reaching, and while there is trend toward curbing smoking within institutions, a great need for empirically-grounded treatments and prevention programs remains.
\end{abstract}

KEYWORDS bealth behaviors, incarcerated populations, smoking bans, smoking cessation, tobacco

Smoking is a major public health problem that is thought to be responsible for over 400,000 deaths each year in the United States (Fisher, Brownson, Heath, Luke, \& Sumner, 2004). As a result of the increased understanding of the dramatic toll smoking takes on our society, prevalence rates have dropped over the last half-century, with 23\% of adults in 2001 identifying themselves as smokers as compared to approximately $42 \%$ in 1965 (U.S. Department of Health and Human Services [DHHS], 1989). These rates vary however, when risk factors are taken into consideration. For example, prevalence rates are higher among low socioeconomic status (SES) groups

The author wishes to thank Erin O'Hea for her teaching, assistance, and critiques during the preparation of this manuscript.

Address correspondence to John J. Donahue, Department of Psychology, La Salle University, 1900 W. Olney Ave., Philadelphia, PA 19141, USA. E-mail: donahuej3@lasalle.edu 
(Centers for Disease Control [CDC], 1999), those with comorbid mental health issues (Hughes, 1993), and other marginalized populations (Belcher, Butler, Richmond, Wodak, \& Wilhelm, 2006). Given these findings, it is not surprising that those incarcerated in prisons and jails have been found to have a much higher rate of cigarette smoking as compared to the general population. Studies across the world have consistently found prevalence rates of smoking within correctional institutions to be approximately 70-83\% (Conklin, Lincoln, \& Tuthill, 2000; Cropsey, Eldridge, \& Ladner, 2004; Belcher et al., 2006; Sieminska, Jassem, \& Konopa, 2006). These alarmingly high numbers are even more startling given that the rates have been found at multiple sites. Furthermore, this is not just a problem confined to adults. While approximately $23 \%$ of high school age children have reported current smoking (CDC, 2006), adolescents involved with the juvenile justice system have reported a $70 \%$ rate of lifetime smoking and a $46.6 \%$ rate of daily smoking (Cropsey, Linker, \& Waite, 2008).

Although the level of smoking among incarcerated populations appears to be at epidemic proportions, the research in this area is limited. The purpose of this paper is to synthesize the existing literature regarding smoking in correctional institutions, identify the direct and indirect effects for both those that are incarcerated as well as society-at-large, and examine what is currently being done to address this issue. Through this review, it is hoped that a clearer understanding can arise as to where the state of the science is, as well as the direction that the field must move in order to combat this important problem.

\section{HEALTH STATUS AND SMOKING BEHAVIORS AMONG PRISONERS}

\section{Health of Prisoners}

Investigations into the overall health behaviors of prisoners and the care that they receive provide some context to the discussion of the smoking behaviors of those incarcerated. The U.S. Department of Justice reports that there are approximately 2.3 million individuals incarcerated in jail and prisons in the United States (2008). This group, which encompasses about $1 \%$ of all adults, appears to be one that is generally much less healthy than the overall population. In a large, representative sample of the entire U.S. prisoner population, Wilper and colleagues (2009) found that over 800,000 inmates reported the presence of at least one chronic medical condition. The authors also found that overall access to medical care in general appeared to be substandard (particularly in jail settings, as compared to prisons), with significant portions of inmates receiving no medical evaluation upon incarceration.

Similarly, Conklin and colleagues (2000), using a large sample of newly incarcerated prisoners housed in a medium-security correctional facility, found that individuals entering the facility had a high rate of current health 
issues, high rates of unhealthy behavior, limited access to health care before entering the facility, and a desire for help in improving their health and health-related behaviors. Both of these studies illustrate that a significant problem exists; individuals that are incarcerated appear to have more health problems, an increased likelihood of engaging in unhealthy behaviors, less treatment before incarceration, and less treatment while incarcerated, but a high desire for healthy change. Given the extremely high rate of smoking among inmates, smoking is believed to be one of the behaviors contributing to this problem (Kauffman, Ferketich, \& Wewers, 2008).

\section{A Smoking Environment}

Consistent with the prevalence rates that have been reported, smoking has long been considered part of the culture within prisons. Social environment plays an important role in the onset and maintenance of smoking (Fisher et al., 2004) and prison is a setting where smoking has generally been viewed as the norm (Afoweso, 2003). Indeed, when risk factors previously described (low SES and higher proportion of mental health issues, for example) are combined with other factors such as a restricted environment that becomes repeatedly associated with smoking and simultaneously provides little else in the way of reinforcement, these high rates of tobacco usage begin to appear less puzzling. Furthermore, the inherently stressful environment of the prison may contribute to the prevalence of smoking as well. Belcher and colleagues (2006) have hypothesized that stressors such as court cases, appeals, recent convictions, and adjustment to prison may all contribute to an increase in smoking among recently admitted inmates. Stressors and the anxiety that they produce have been identified as reasons to smoke among prisoners in other studies as well (Baker et al., 2006; Richmond et al., 2006; Sieminska et al., 2006).

Additionally, cigarettes are often used as barter within correctional institutions, where black market cigarette economies are often found. Partly as a result of this, there can be an increased frequency of smoking hand-rolled cigarettes, which is even worse for one's health given their increased tar and nicotine contents (Darrall \& Figgins, 1998). When considering this in conjunction with the finding that correctional inmates smoke similar rates of cigarettes per day as compared to community samples, it appears that incarcerated smokers may be at an elevated risk to become dependant on the physiological effects of smoking and have a higher likelihood of developing later health problems as a result (Belcher et al., 2006).

\section{Smokers' Perceptions}

As smoking has been viewed as a sociocultural norm of prison life, it is important to examine the research investigating the knowledge, attitudes, 
and expectancies of those that are incarcerated. Partly as a result of this cultural perception, efforts to restrict smoking behaviors within prisons have been met with arguments that these restrictions entail a form of inhumane treatment through the deprivation of a basic joy (Belcher et al., 2006). However, while the question of what constitutes inhumane treatment is beyond the scope of this review, the actual perceptions of prisoners themselves are indeed an important area of discussion. Cropsey et al. (2004) investigated this as part of a larger study on the smoking behaviors of female inmates and found that $49.9 \%$ of their sample wanted to quit smoking "very much," and $64.2 \%$ indicated interest in taking part in a smoking cessation program if offered.

Similarly, an investigation of prisoners in Poland, a country that has historically had one of the higher smoking rates in the world, found that $75 \%$ of smokers attempted to quit in the past (Sieminska et al., 2006). Moreover, smokers in this study demonstrated limited knowledge on the deleterious effects of cigarettes, with $28 \%$ of smokers with higher educational achievement recognizing the negative impacts on health as compared to only $19 \%$ of those with a basic education. While the data on prisoners' perceptions and knowledge about smoking are considered limited in scale, the investigations that have been conducted do appear to reflect a group that generally wants to quit, has tried quitting in the past, and may benefit from education regarding the detrimental effects of smoking.

\section{FACTORS ASSOCIATED WITH SMOKING IN PRISONS}

\section{Health Consequences}

That cigarette smoking is a health problem currently facing the correctional system has already been established, but the specific effects of this phenomenon have not yet been delineated. Of the serious medical problems reported by prisoners, most were smoking related, such as cardiovascular, circulatory, respiratory, kidney, and liver problems (Maruschak \& Beck, 2001). Smoking has also been linked to decreased oral health among prisoners. One recent study found that among inmates, those that smoked had the poorest oral health, with more missing teeth and less tooth fillings (Cropsey, Crews, \& Silberman, 2006). At the same time, smoking has been associated with increased dental caries in a sample of female federal prisoners (Heng, Badner, \& Freeman, 2006).

\section{Air Quality}

In addition to the negative effects on the smokers themselves, correctional facilities that still allow indoor smoking can be harmful for others as well. Although the tobacco policies in prisons have become more restricted over 
the years, the increase in indoor smoking bans has been a relatively recent phenomenon (Kauffman et al., 2008). This is unfortunate given that the health risks associated with exposure to secondhand smoke include the development of heart disease and lung cancer, among others (Proescholdbell, Foley, Johnson, \& Malek, 2008). In light of these circumstances, research in this area has looked at the air quality of correctional institutions with and without smoking bans. An example of this comes from a study that gathered air samples from a prison with no smoking restrictions indoors, then again after tobacco control policies were instituted (Hammond \& Emmons, 2005). Air quality improved after the restrictions were implemented however before that, these investigators found the nicotine concentration of most sleeping areas to be about five times the average nicotine concentration of a smoker's home, with one large bunkroom exhibiting a concentration 12 times that of a typical smoker's home. These results illuminate the fact that indoor smoking in correctional facilities can contribute to an unhealthy environment where the likelihood for passive smoke inhalation is high. In line with this, an investigation into the air quality of prisons in North Carolina prior to the enactment of a smoking ban demonstrated that levels of respirable suspended particulates (a marker that is accepted as a measure of secondhand smoke exposure) decreased $77 \%$ after the institution of a smoking ban (Proescholdbell et al., 2008).

While the topic of smoking bans will be discussed in further detail later, it appears that restricting smoking indoors at prisons should be standard practice given the environmental impact and adverse health consequences that may arise if no restrictions are in place. Consistent with the general literature on smoking and secondhand smoking, tobacco usage among correctional inmates has been linked to increased occurrences of a variety of medical problems and the creation of an aversive environment not conducive to healthy living. Overall, the prevalence of smoking in these settings appears to partly explain the high rate of health problems among the entire correctional population, even when considering those inmates that do not smoke.

\section{Available Resources}

Unfortunately, health care problems within correctional institutions may be a problem that could continue to worsen. As the result of a variety of factors such as mandatory sentencing laws, the aging of the baby boomers, and an overall increase in lifespan, older prisoners are a quickly rising population (Aday, 2003; Loeb \& Steffensmeier, 2006). Although there is evidence that nonsmoking inmates are significantly older than their smoking counterparts (Belcher et al., 2006), this highlights another pertinent issue: prison health services. Older prisoners constitute major users of health care resources in prisons; however one in six prisoners under age 50 report a serious medical 
problem as well (Cropsey et al., 2004). These problems are often smoking related. With a quickly growing aging population and younger cohorts suffering smoking-related illnesses, a burden is sure to continue to be felt by the providers of health care services. Inmate health care consisted of $9.8 \%$ of overall prison budgets in 1999 , with the annual per capita cost at $\$ 2,248$ (a number that has been steadily rising from \$906 in 1982) (Hegamin, Longshore, \& Monahan, 2002). The economic impact of this problem is significant in that if health care costs continue to rise; this will result in the losses or reductions of other prison services.

In the medical community, an increased emphasis has been placed on the concept of preventative medicine, where efforts are made to take proactive efforts to prevent illness rather than reactively treat it later (Chang \& Nash, 1998). While this is likely an effective direction if one wants to reduce the rate of illness and address the financial burden of rising healthcare costs, one review of prison health care in the United Kingdom concluded that an underlying theme exists across prisons that while health promotion is thought to be considered essential, it is underused and perhaps not well understood (Watson, Stimpson, \& Hostick, 2004). However, these authors did note that health promotion in prisons is still early in its history, thus the focus should be on instituting and tracking practices in this area.

\section{Summary of Problem}

The literature on the effects of cigarette smoking within the correctional system reveals that this is a problem that impacts the immediate environment and all those that are in it, contributing to the overall poor health of those that are incarcerated. It is an economic burden as well, given the rising rates of health care costs for those that are incarcerated. These effects are both immediate and long-term, micro and macro. And the problem is not just confined to the prison environment, as while prison may appear similar to one on its face, life here does not occur in a vacuum. The vast majority of these individuals are released back into the community and improved treatment of conditions (and increases in health promotion behaviors) can have implications for community health in general (Wilper et al., 2009). Wilper and colleagues have indicated that addressing poor physical conditions and decreasing one's likelihood for developing further conditions can assist in both reducing health care disparities and facilitating the likelihood that one successfully reintegrates into the community. If this does not occur, or worse, one's health deteriorates while incarcerated, the problem continues to perpetuate and the question again arises as to what the purpose of prison actually is, a consequence that may result in rehabilitation, or simply mere retribution. This brings us to the crux of the matter, what is actually being done to address cigarette smoking within prisons? 


\section{SMOKING RESTRICTIONS WITHIN PRISONS}

In an attempt to curb the detrimental effects of cigarette smoking in prisons, numerous agencies have begun placing restrictions on where tobacco usage is allowed. Given that smoking has long been considered a normality of prison life, with many instances of cigarettes being distributed to inmates freely by both institutional authorities and other organizations (Awofeso, 2003), it is not surprising that prisons have lagged behind others in the restriction of indoor smoking. This change did get underway though in the 1990s and there have been large increases in these restrictions during recent years. Kauffman and colleagues (2008) investigated prison tobacco policies in a survey of 52 U.S. Departments of Corrections and found that $60 \%$ currently have total bans on prison grounds and an additional $27 \%$ have an indoor ban on tobacco usage. This is a dramatic change from the 1980s, where a survey of 19 correctional systems found zero smoke-free living areas and $53 \%$ of those surveyed distributed free tobacco to inmates (Romero \& Connell, 1988). Thus, it appears that while the prevalence rates still reflect a high level of smoking among prisoners, there is a move away from indoor smoking that will help minimize the negative effects that occur when exposed to secondhand smoke. With this said, the survey conducted by Kauffman et al. obtained data from state and federal correctional administrations; tobacco policies in local jails were not gathered in this study. This is important to note as jails (which are different from prisons in that they generally house inmates awaiting trial or those with brief sentences) are predominantly run by local counties (or in some cases, private corporations), and their policies may be different than those of the state and federal prison systems.

The National Network on Tobacco Prevention and Poverty (NNTPP; Chavez et al., 2003) conducted a survey of tobacco policies in prisons and jails in which data from 48 jails were obtained. While over $70 \%$ of those that responded reported tobacco-free policies, the authors reported that there appears to be some question as what "tobacco-free" actually means (for instance, the majority of the policies affected just inmates, rather than inmates and staff). Overall, because of the limited data on current tobacco policies in local jails, this remains an important area for future investigation. Generally though, the current survey data of correctional institutions do reflect a trend of greater restriction with regard to the use of tobacco on prison grounds.

\section{DO RESTRICTIONS HELP SMOKERS?}

\section{Short-Term Effects}

In the survey on prison tobacco policies conducted by Kauffman and colleagues (2008), correctional authorities identified the health and safety 
of prisoners/employees, complaints and litigation regarding secondhand smoke, and compliance with legislation as common reasons for implementing smoking restrictions. Given that health and safety was the most frequently cited reason provided (69.4\% of responses), it is important to evaluate the influence that these bans and restrictions have on actual smoking behavior. Cropsey and Kristeller (2005) examined this very issue in a study assessing levels of distress, nicotine dependence, and cravings of prisoners both before a smoking ban was implemented, and at two points after the restrictions was put in place. Results of this investigation indicated that $76 \%$ of subjects continued to smoke even after the smoking ban was implemented. This is a startlingly high number, especially when considering that admitting to engaging in a prohibited behavior in a prison may be affected by socially desirable responding. In an attempt to reconcile these findings, the authors directed attention to a study conducted by Carpenter, Hughes, Solomon, and Powell (2001), in which a survey of prison employees found that only $15 \%$ of employees who smoke favored a ban on inmate smoking. As such, it is possible that aggressive enforcement of cigarette smoking bans may not occur because of employees' low motivation to enforce a guideline that they fundamentally disagree with (Cropsey \& Kristeller, 2005). These authors' findings provide preliminary evidence as to the effectiveness of smoking bans on actual smoking behaviors and replication is necessary to gain a better understanding as to the generalizability of these results. Also, given that the effectiveness of a smoking ban is likely to be largely dependant on the level of enforcement of that ban, future investigations into employee enforcement of smoking bans are needed as well.

\section{Long-Term Effects}

While individual differences in smoking ban enforcement are bound to occur, it is likely that one will find many correctional institutions, especially those that have had bans in effect for longer periods, where guidelines are more strictly enforced. In these cases, bans and restrictions should greatly decrease the smoking behaviors of prisoners within those facilities. But does this actually result in long-term behavior change? In a study examining the predictors of current need to smoke among prisoners incarcerated in a facility with a smoking ban, Voglewede and Noel (2004) found that future intention to smoke was a significant predictor of current need to smoke in this sample. In this regard, prisoners that are experiencing a high level of cigarette cravings when introduced to a nonsmoking environment (i.e., prison with a ban in place) may be more likely to return to their normal rates of smoking when they are released from incarceration. As such, smoking cessation programs that target these cravings may be beneficial for this group of individuals who appear to be at high risk for later relapse. Indeed, there is data to indicate that smokers incarcerated in institutions with smoking bans 
do not continue to abstain from smoking once they are released. Specifically, a survey of individuals that were previously held in a county correctional institution with a smoking ban found that only $37.3 \%$ did not smoke on their first day of release and just $2.4 \%$ continued to refrain from smoking six months after release (Tuthill et al., 2002). Similarly, results from Chavez and colleagues' 2003 survey indicate that correctional administrators' estimates of the percentage of inmates who return to smoking upon release range from $76 \%$ to $100 \%$. Collectively, this evidence appears to demonstrate that while tobacco bans may have short-term effects of reducing smoking in correctional facilities, they are not useful in reducing intentions to quit or in effecting long-term behavior change. Forced abstinence is not enough; there must be programs geared toward smoking cessation as well. However, in the Kauffman et al. survey (2008), only $39 \%$ of prisons that enacted a total tobacco ban continued to offer smoking cessation programs and just 35\% made tobacco cessation aids available. These rates are disappointing in that they appear to reflect a mentality that restriction alone is a sufficient response to the problem.

\section{SMOKING CESSATION PROGRAMS IN CORRECTIONAL INSTITUTIONS}

The literature on smoking cessation generally indicates that extended and comprehensive treatments, which are tailored to the client and include behavioral components, increase the likelihood of successful cessation (Fisher et al., 2004) and the resources necessary to make these intensive interventions work result in benefits that are cost-effective (Warner, 1997). However, while most prison systems in the United States that have not implemented a total smoking ban have reported that tobacco cessation programs are present in their facilities (Kauffman et al., 2008), another survey that has included jails in their samples found that $80 \%$ of correctional institutions surveyed did not have these programs (Chavez et al., 2003). Furthermore, correctional administrators in this same study identified smoking cessation programs as low priority as compared to other health care issues. This is unfortunate given the overall ill health of prisoners in general, the adverse effects that cigarette smoking is associated with in prisoners and in the broader population, and the unique opportunity incarceration provides with regard to interventions (in that it allows for an opportunity to address the health needs of at-risk populations). One possible factor contributing to prison administrators' diminished focus on smoking cessation programs may be their perceived effectiveness. While this is only speculation at this time, if these programs are viewed as ineffective, then it stands to reason that they would be given low priority and attempts to reduce smoking would instead by directed toward restrictive policies. 
Through the implementation of effective smoking cessation programs, a change may occur with regard to their perceived importance. However, while cessation programs are in place at numerous facilities, a search of the literature revealed just one randomized clinical trial with this population that could be used to facilitate future treatment planning (Cropsey et al., 2008). This stands as an important area for future research, as several factors unique to the prisoner's experience are likely to influence treatment, and these factors should be taken into consideration when designing an effective program. While it appears that just one clinical trial has been conducted, there are some published studies on specific smoking cessation programs implemented in correctional institutions.

\section{Early Research}

Published research evaluating interventions for smoking behaviors within correctional environments are rare. Through reviewing the literature that is available on this subject, the reader will see that while the limited data does provide encouraging results, the paucity of investigations and methodological drawbacks of some of the studies highlight the need for continued research in this area. In the 1970s, investigators examined the effectiveness of a group-based smoking cessation program utilizing self-control techniques in a sample of federal prisoners (Edinger, Nelson, Davidson, \& Wallace, 1978). These authors implemented two studies and found that some cigarette smoking reductions did occur, some components (such as mini-goal setting) were more helpful than others, and using a contracting procedure (during the second study) appeared to decrease the likelihood of subject dropout. However, these two studies both have severe methodological limitations in that a high level of attrition plagued Study 1, follow-up periods were relatively short, and neither study included a control group. With this being said, this research should be considered a valuable endeavor as Edinger and colleagues demonstrated that some modification of smoking behaviors can occur in prison settings during an era where smoking was still very much a part of the culture and prison smoking bans were virtually nonexistent.

\section{Current Programs}

In an effort to find current research on smoking cessation programs within prisons, we turn to Australia, where significant attempts are underway to address this problem. While indoor restrictions have begun to take shape in Australia, they do not appear to be as far reaching as in the U.S., as the view that this constitutes a form of inhumane treatment is more prevalent in that country (Belcher et al., 2006). Given this perspective, a greater emphasis has been placed on prison-based tobacco control programs, as they are 
commonly referred (Awofeso, 2003). An effective program of this sort has been developed by Awofeso and colleagues (Awofeso, Levy, \& Morris, 2001; Awofeso, 2003) through the usage of a social marketing concept approach. Awofeso argues that in order for smoking cessation programs to be successful in prisons, change must occur at two levels, "(a) prison culture and prison policies that tend to encourage tobacco use, and (b) behavior change of smoker inmates such that a non-smoking status is perceived as achievable, worthwhile, healthy, and socially desirable" (2003, p. 121). Social marketing has been defined as "the application of commercial marketing technologies to the analysis, planning, execution, and evaluation of programs designed to influence voluntary behavior of target audiences in order to improve their personal welfare and that of society" (Andreasen, as quoted in Awofeso, 2003, p. 122). According to the author, this approach may facilitate the effectiveness of smoking cessation programs by framing smoking as a behavior rather than a "chronic disease" or addiction; increasing advocacy for healthy custodial policies regarding tobacco in prisons; increasing awareness of both the consequences of smoking and the benefits of cessation programs; increased analysis of issues and objectives within the programs; and by addressing issues that are specific to prisoners with high smoking rates (Awofeso, 2003). While a more detailed description of the social marketing approach to smoking cessation is beyond the scope of this article, the reader is referred to Awofeso (2003) for a thorough review of its framework. Additionally, although this program has been implemented in several prisons in Australia, empirical research evaluating this approach in comparison to other approaches could not be located. This remains as an important area for further research.

A recent study, also out of Australia, examined the feasibility of a multi-component smoking intervention for prison inmates, which included brief cognitive-behavioral therapy, nicotine replacement therapy, and a non-nicotine pharmacotherapy (bupropione hydrochloride sustained release) (Richmond et al., 2006). This pilot study resulted in more than one quarter of the inmates who began treatment, maintaining abstinence at six-month follow-up (biochemically validated). While lower than community studies utilizing this approach, the authors noted that these results are encouraging given influencing factors such as the stressors of prison life, the smoking culture in prisons, subjects' histories of comorbid substance abuse, and high levels of nicotine dependence (Richmond et al., 2006). Additionally, a very important finding in this study was that $95 \%$ of those inmates who relapsed during the trial reported that they were willing to attempt to quit again using this treatment approach. Overall, although the Richmond et al. trial also suffers from methodological limitations such as the lack of a control group and small sample size, it is an important preliminary finding regarding the effectiveness of community smoking cessation programs in prison contexts. 


\section{A Step Forward}

Finally, a recent investigation by Cropsey and colleagues (2008) investigated the effects of a smoking cessation program, which combined a behavioral and pharmacologic intervention, among female prisoners in the United States. This study is notable in that it represents the first known prison-based smoking cessation program evaluated using a randomized controlled trial with control group design. Results indicate that the combined behavioral and pharmacologic intervention was efficacious as compared to wait list controls in promoting smoking cessation (quit rates of $14 \%$ vs. $2.8 \%$ at six-month follow-up) (Cropsey et al., 2008). The outcome of this study was similar to those found among community samples, demonstrating that effective smoking cessation programs are feasible in prison settings. Given that this study is the first randomized controlled trial testing a smoking intervention among inmates, and that it only focused on female inmates, continued research of this type is important so that the generalizability of results can be evaluated.

While the research on prison-based smoking cessation programs provides evidence that smokers in these contexts can benefit from interventions, the limited literature in this area is quite unfortunate given the scope of the problem. Kauffman et al. (2008) found in their survey that cessation programs are prevalent in the U.S., however, given the few intervention studies covered in this review, critical evaluations of these programs have rarely appeared in the literature. As such, there is a need for additional methodologically sound outcome studies in prison settings, utilizing treatment approaches that have been found to be efficacious in community samples.

\section{CONCLUDING REMARKS AND FUTURE DIRECTIONS}

The U.S. Department of Health and Human Services has identified cigarette smoking as the "single most important preventable cause of premature death" (U.S. DHHS, as quoted in Fisher et al., 2004, p. 77). This understanding of the negative effects of smoking has greatly helped public health efforts at reducing the amount of smokers in our society. However, prevalence rates among the prison population remain at staggeringly high levels (Conklin et al., 2000; Belcher et al., 2006; Cropsey et al., 2004). The purpose of this review was to organize the extant literature on cigarette smoking behaviors among incarcerated populations, summarize the nature of the problem, and examine what the responses are from the health care community, the prison industry, and society at-large. Evidence suggests that prisoners as a group suffer from higher rates of physical problems and illnesses as compared to the community, with much of these problems seen as smoking-related (Maruschak \& Beck, 2001). However a strong "culture of smoking" within prisons may have contributed to a slower response addressing this issue, even with mounting evidence of the detrimental consequences for both 
the inmates themselves (Cropsey et al., 2006; Proescholdbell et al., 2008; Belcher et al., 2006), as well as the overall economic impact (Hegamin et al., 2002). In the U.S., the response to this has been largely in the form of increased restrictions on tobacco usage on prison grounds (Kauffman et al., 2008), however the effects these bans have on continued abstinence both long-term (Tuthill et al., 2002) and short-term (Cropsey \& Kristeller, 2005) are not reassuring. Finally, while investigations into the efficacy and effectiveness of smoking interventions are emerging, the literature in this area is still in its infancy. Only three outcome studies could be located in this review (Edinger et al., 1978; Richmond et al., 2006; Cropsey et al., 2008), and just one (Cropsey et al., 2008) included in its methods randomization and the use of a control group.

It is evident that there is much work to be done in response to the problem of tobacco usage among those that are incarcerated. Additional welldesigned outcome studies are sorely needed so that the health care providers and prison administrators can have a clearer understanding of what works and with whom. In line with this, investigations examining the factors uniquely associated with prisoners (i.e., prison stressors, transfers to other facilities, conflicts between treatment strategies and prison policies), and how these factors moderate the relationship between intervention and outcome are necessary as well. Indeed, these moderators are recognized by Awofeso's social marketing approach (2003), as well as Richmond and colleagues (2006), and it is likely that interventions that fail to address these factors will not reach their full potential. Another suggested line of research is to examine the effectiveness of smoking cessation programs in conjunction with the implementation of smoking restrictions that are ever increasing. Given the limited effectiveness smoking bans have had on continued abstinence, this review echoes the call from others to offer evidence-based smoking cessation programs along side of tobacco bans or restrictions.

Finally, as it has in the larger community, public health efforts geared toward prevention should be evaluated within a correctional context. Although it is not known how many smokers begin smoking after incarceration, there is evidence that substantial numbers of prisoners started smoking after entering prison (Cropsey et al., 2008). One possible avenue of research may include the examination of a smoking prevention project similar to the Jailbreak Health Project (Minc, Butler, \& Gahan, 2007). This project, implemented in Australia, includes a weekly radio program composed of opinion pieces, music, and poetry from people involved in the criminal justice system. Also included in this program, are a number of health promotion messages that are directed toward the prison population. This strategy, which may be a useful way to complement written health promotion materials because of the commonly poor literacy levels found among prisoners, provides information to prisoners regarding sexual health and diseases. Programs such as the Jailbreak Health Project may be modified to include 
tobacco education and could prove beneficial with regard to increasing prisoners' awareness about the negative consequences of smoking and help facilitate their motivation to change.

While somewhat paradoxical in that prisons are generally thought of as a place for those "too dangerous for society," our correctional systems are composed of some of the population's more vulnerable individuals. These at-risk individuals often have little or no previous access to health care services and the "unique opportunity offered to jails is that they can reach vulnerable populations not usually served by other systems and engage them in short-term health interventions" (Hammett, Gaiter, \& Crawford, 1998, p. 100). Unfortunately, a convergence of factors such as fixed sentencing and the aging of the prison population are negatively impacting the fiscal and medical structure of correctional health care. Directing our attention toward the monumental problem of cigarette smoking is an efficient and effective means of addressing this problem in our prisons. While the extent of the problem may be apparent, there is still much work to be done in the area of treatment and prevention research.

\section{REFERENCES}

Aday, R. J. (2003). Aging prisoners: Crisis in American corrections. Westport, CT: Praeger.

Awofeso, N. (2003). Implementing smoking cessation programmes in prison settings. Addiction Research \& Theory, 11(2), 119-130.

Awofeso, N., Levy, M., \& Morris, S. (2001). Managing a tobacco control program in NSW correctional centers, 1999-2001. NSW Public Health Bulletin, 12, 193-195.

Baker, A., Ivers, R. G., Bowman, J., Butler, T., Kay-Lambkin, F. J., Wye, P., et al. (2006). Where there's smoke, there's fire: High prevalence of smoking among some sub-populations and recommendations for intervention. Drug and Alcohol Review, 25(1), 85-96.

Belcher, J. M., Butler, T., Richmond, R. L., Wodak, A. D., \& Wilhelm, K. (2006). Smoking and its correlates in an Australian prisoner population. Drug and Alcohol Review, 25, 343-348.

Bureau of Justice Statistics, U.S. Department of Justice. (2008). Prison statistics, summary findings. Retrieved April 2, 2009, from http://www.ojp.usdoj.gov/ bjs/prisons.htm

Carpenter, M. J., Hughes, J. R., Solomon, L. J., \& Powell, T. A. (2001). Smoking in correctional facilities: A survey of employees. Tobacco Control, 10(1), 38-42.

Centers for Disease Control. (1999). Tobacco use-United States, 1900-1999. Morbidity and Mortality Weekly Reports, 48, 986-993.

Centers for Disease Control. (2006). Cigarette use among high school studentsUnited States, 1991-2005. Morbidity and Mortality Weekly Reports, 55, 724-726.

Chang, K., \& Nash, D. (1998). The role of pharmacoeconomic evaluations in disease management. Pharmacoeconomics, 14(1), 11-17. 
Chavez, R. S., Oto-Kent, D. S., Porter, J., Brown, K., Quirk, L., \& Lewis, S. (2003). Tobacco policy, cessation, and education in correctional facilities. Retrieved March 29, 2009, from http://www.healthedcouncil.org/breakfreealliance/pdf/ ncchc.pdf

Conklin, T. J., Lincoln, T., \& Tuthill, R. W. (2000). Self-reported health and prior health behaviors of newly admitted correctional inmates. American Journal of Public Health, 90, 1939-1941.

Cropsey, K., Crews, K. M., \& Silberman, S. L. (2006). Relationship Between Smoking Status and Oral Health in a Prison Population. Journal of Correctional Health Care, 12, 240-248.

Cropsey, K., Eldridge, G. D., \& Ladner, T. (2004). Smoking among female prisoners: An ignored public health epidemic. Addictive Behaviors, 29(2), 425-431.

Cropsey, K., Eldridge, G., Weaver, M., Villalobos, G., Stitzer, M., \& Best, A. (2008). Smoking cessation intervention for female prisoners: Addressing an urgent public health need. American Journal of Public Health, 98, 1894-1901.

Cropsey, K., \& Kristeller, J. L. (2005). The effects of a prison smoking ban on smoking behavior and withdrawal symptoms. Addictive Behaviors, 30, 589-594.

Cropsey, K., Linker, J. A., \& Waite, D. E. (2008). An analysis of racial and sex differences for smoking among adolescents in a juvenile correctional center. Drug and Alcohol Dependence, 92(1-3), 156-163.

Darrall, K. G., \& Figgins, J. A. (1998). Roll-your-own smoke yields: Theoretical and practical aspects. Tobacco Control, 7, 168-175.

Edinger, J. D., Nelson, W. M., Davidson, K. M., \& Wallace, J. (1978). Modification of smoking behaviors in a correctional institution. Journal of Clinical Psychology, 34, 991-998.

Fisher, E. B., Brownson, R. C., Heath, A. C., Luke, D. A., \& Sumner, W., II. (2004). Cigarette smoking. In J. M. Raczynski \& L. C. Leviton (Eds.), Handbook of clinical bealth psychology: Volume 2. Disorders of behavior and health (pp. 75-120). Washington, DC: American Psychological Association.

Hammett, T. M., Gaiter, J. L., \& Crawford, C. (1998). Reaching seriously at-risk populations: Health interventions in criminal justice settings. Health Education E Behavior, 25(1), 99-120.

Hammond, S. K., \& Emmons, K. M. (2005). Inmate exposure to secondhand smoke in correctional facilities and the impact of smoking restrictions. Journal of Exposure Analysis and Environmental Epidemiology, 15, 205-211.

Hegamin, A., Longshore, D. L., \& Monahan, G. (2002). Health services in correctional settings: Emerging issues and model programs. In C. G. Leukefeld, F. M. Tims, \& D. Farabee (Eds.), Treatment of drug offenders: Policies and issues (pp. 335347). New York: Springer Publishing Co.

Heng, C. K., Badner, V. M., \& Freeman, K. D. (2006). Relationship of cigarette smoking to dental caries in a population of female inmates. Journal of Correctional Health Care, 12, 164-174.

Hughes, J. R. (1993). Possible effects of smoke-free inpatient units on psychiatric diagnosis and treatment. Journal of Clinical Psychiatry, 54(3), 109-114.

Kauffman, R. M., Ferketich, A. K., \& Wewers, M. E. (2008). Tobacco policy in American prisons, 2007. Tobacco Control, 17, 357-360. 
Loeb, S. J., \& Steffensmeier, D. (2006). Older male prisoners: Health status, self-efficacy beliefs, and health-promoting behaviors. Journal of Correctional Health Care, 12, 269-278.

Maruschak, L. M., \& Beck, A. L. (2001). Medical problems of inmates, 1997 [No. NCJ 181644]. Washington, DC: U.S. Department of Justice, Bureau of Justice Statistics.

Minc, A., Butler, T., \& Gahan, G. (2007). The jailbreak health project: Incorporating a unique radio programme for prisoners. International Journal of Drug Policy, 18, 444-446.

Proescholdbell, S. K., Foley, K. L., Johnson, J., \& Malek, S. H. (2008). Indoor air quality in prisons before and after implementation of a smoking ban law. Tobacco Control, 17, 123-127.

Richmond, R. L., Butler, T., Belcher, J. M., Wodak, A., Wilhelm, K. A., \& Baxter, E. (2006). Promoting smoking cessation among prisoners: Feasibility of a multi-component intervention. Australian and New Zealand Journal of Public Health, 30, 474-478.

Romero, C. A., \& Connell, F. A. (1988). A survey of prison policies regarding smoking and tobacco. Journal of Prison and Jail Health, 7, 27-36.

Sieminska, A., Jassem, E., \& Konopa, K. (2006). Prisoners' attitudes towards cigarette smoking and smoking cessation: A questionnaire study in Poland. BMC Public Health, 6(181), 1-9.

Tuthill, R. W., Lincoln, T., Conklin, T. J., Kennedy, S., Hammett, T. M., \& Roberts, C. A. (2002, October). Does involuntary cigarette smoking abstinence among inmates during correctional incarceration result in continued abstinence post release? Poster presented at the National Conference on Correctional Health Care, Nashville, TN.

U.S. Department of Health and Human Services. (1989). Reducing the health consequences of smoking - 25 years of progress. A report of the Surgeon General [DHHS publication (CDC) 89-8411]. Rockville, MD: U.S. Department of Health and Human Services, Public Health Services, Centers for Disease Control, Center for Chronic Disease Prevention and Health Promotion, Office on Smoking and Health.

Voglewede, J. P., Jr., \& Noel, N. E. (2004). Predictors of current need to smoke in inmates of a smoke-free jail. Addictive Behaviors, 29, 343-348.

Warner, K. E. (1997). Cost-effectiveness of smoking-cessation therapies: Interpretation of the evidence and implications for coverage. Pharmacoeconomics, 11, 538-549.

Watson, R., Stimpson, A., \& Hostick, T. (2004). Prison health care: A review of the literature. International Journal of Nursing Studies, 41, 119-128.

Wilper, A. P., Woolhandler, S., Boyd, J. W., Lasser, K. E., McCormick, D., Bor, D. H., \& Himmelstein, D. U. (2009). The health and health care of US prisoners: Results of a nationwide survey. American Journal of Public Health, 99, 666-672. 